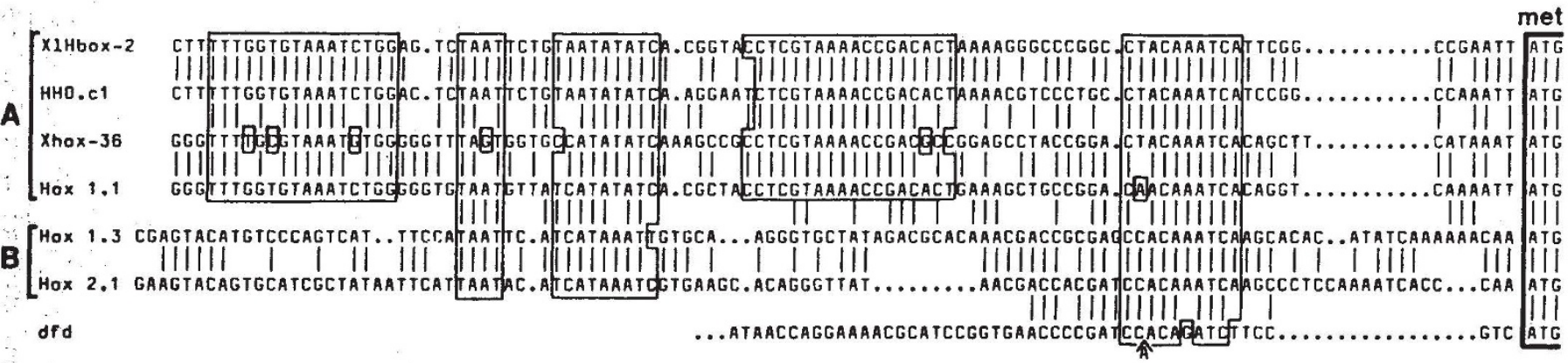

Conserved regions in the $5^{\prime}$-non-coding regions in homoeobox-containing genes. The initiator AUG is at the right-hand end of the figure. Vertical lines between pairs of sequences indicate identical nucleotides. Regions of high conservation are boxed. A short region conserved in the Drosophila gene Deformed is indicated. Fushi tarazu has a small stretch of nucleotides (TCTGATTTTGCTATATAT, approximately-100 upstream of the AUG) similar to the second and third boxed regions of X1Hbox 2 and HHO.cl (not shown).

Most interestingly, we unexpectedly found extensive nucleotide conservations in the $5^{\prime}$-non-coding regions immediately preceding the initiator AUG, as shown in the figure. The conservation is maximal among the four genes mentioned above. For X1Hbox 2 (ref. 3) and HHO.cl (ref. 9) it is $91 \%$ (over 99 nucleotides), for Xhox36 (ref. 2) and Hox 1.1 (ref. 7) it is $77 \%$ (over 102 nucleotides), and for Hox 1.1 and $\mathrm{X} 1 \mathrm{Hbox} 2$ it is $77 \%$ (over 100 nucleotides). Sequence conservations in this region occur in other genes: mouse Hox 2.1 (ref. 5) and Hox 1.3 (ref. 6) are $63 \%$ conserved over 114 nucleotides.

Why are non-coding nucleotides conserved in many genes from such diverse organisms? Perhaps this merely reflects the common evolutionary origin of homoeobox genes. Alternatively, the sequences may have remained invariant because of strong evolutionary pressure to preserve a common function. The best argument in favour of this is provided by the homologues X1Hbox 2 and HHO.cl (ref. 9). These two genes have been evolving for at least 350 million years, since the separation of amphibians and the mammalian ancestors. Their 5'-noncoding regions next to the AUG are $91 \%$ conserved, but the 3 '-non-coding regions show no sequence conservation.

In the coding region between the amino terminus and the homoeobox there is very little nucleotide similarity and even in the homoeobox regions, where the protein reading frame is under strong pressure to remain invariant, the conservation is only $81 \%$. We think the $5^{\prime}$-non-coding region is the most conserved not only because of a common evolutionary origin but because it carries an important biological function.

The conservations shown in the figure are not due to a translational reading frame, because in all sequences there are stop codons in all reading frames and frequent insertions that would produce frameshifts. As the 5'-leader conservations are closely associated with the initiation codon, an attractive hypothesis is that these sequences are involved in translational control. Translational control has been implicated in the expression of homoeobox genes in Drosophila: the best example being the maternal gene caudal whose mRNA, although uniformly distributed in the egg, is translated only in the posterior of the embryo ${ }^{11.12}$. The possibility of translational regulation has been shown for the frog gene X1Hbox 2 in an experiment in which deletion of most of the 5 '-leader of a cDNA clone, leaving only 26 nucleotides in front of the AUG, stimulated in vitro translation of SP6 messenger RNAs over 20 -fold by both reticulocyte and wheat-germ systems ${ }^{4}$. The proteins translated from both constructs were of the same size ${ }^{4}$, providing direct evidence that the $5^{\prime}$-conserved region is not translated.

ThOMAs R. BürgLIN Christopher V.E. Wright EDDY M. DE ROBERTIS

Department of Biological Chemistry,

University of California,

Los Angeles, California 90024-1737, USA

1. Harvey, R.P., Tabin, C.J. \& Melton, D.A. EMBO J. 5 1237-1244 (1986)

2. Condie, B.G. \& Harland, R.M. Development 101, 93-106 (1987).

. Wright, C.V.E., Cho, K.W.Y., Fritz, A., Bürglin, T. \& De Robertis, E.M. EMBOJ. (in the press)

4. Fritz, A. \& De Robertis, E.M. Nucleic Acids Res. (in the press).

5rumlauf, R., Holland, P.W., McVey, J.H. \& Hogan B.L.M. Development 99, 603-617 (1987)

6. Odenwald, W.F. et al. Genes Dev. 1, 482-496 (1987).

7. Kessel, M., Schulze, F., Fibi, M. \& Gruss, P Proc. natn. Acad. Sci. U.S.A. 84, 5306-5310(1987).

8. Mavilio, F. et al. Nature 324, 664-668 (1986).

Simeone, A. et al. Proc. natn. Acad. Sci. U.S.A.84, 4914 4918 (1987).

10. Meijlink, F. et al. Nucleic Acids Res. 15, 6773-6786 (1987) 11. Mlodzik, M \& Gehring, W.J. Cell 48, 465-478 (1987)

12. MacDonald, P.M. \& Struhl, G. Nature 324, 537-54 (1986).

\section{AIDS incubation period in male haemophiliacs}

SIR-Ekert ${ }^{1}$ concludes that there is no adequate explanation for the tenfold lower rate of conversion of infection with the human immunodeficiency virus (HIV) to clinical AIDS (acquired immune deficiency syndrome) in male haemophiliacs compared with other groups of HIV carriers. A possible explanation is that, unlike heterosexual male haemophiliacs, other groups of HIV carriers are exposed during sexual intercourse to the immunosuppressant effects of seminal plasma.

Human seminal plasma contains various immunosuppressive agents ${ }^{2}$. In par- ticular, seminal plasma may inhibit the normal immune response to a viral infection $^{3}$. Prostaglandin $E_{2}$, a constituent of seminal plasma, has been shown in vitro to facilitate HIV replication ${ }^{4}$. Clinically, both the transmission ${ }^{5}$ and the neoplastic complications ${ }^{6}$ of HIV are associated with receptive anal intercourse. We have recently suggested that following sexual intercourse the suppression of the immune response to HIV by seminal plasma may be important in patients who are already HIV carriers ${ }^{3}$. Such an effect could hasten the development of clinical AIDS in HIV carriers and is consistent with the higher incidence of Kaposi's sarcoma ${ }^{6}$ and lymphomas ${ }^{7}$ in male homosexual HIV carriers compared with other HIV carriers, including haemophiliacs.

Thus, a longer incubation period and a lower incidence of clinical AIDS in haemophiliacs following HIV infection may be more apparent than real because of the shorter incubation period in other HIV carriers.

\section{M.J. TURNER J.O. White}

W.P. SOUTTER

Institute of Obstetrics and Gynaecology,

Royal Postgraduate Medical School,

Du Cane Road, London W12 OHS, UK

1. Ekert, H. Nature 329, 494 (1987)

2. Alexander, N.J. \& Anderson, D.J. Fertil. Steril. 47, 192-205 (1987)

Turner, M.J., White, J.O. \& Soutter, W.P. Immun. Today 8, 258 (1987)

4. Kuno, S. et al. Proc. natn. Acad. Sci. U.S.A. 83, 3487-3490 (1986)

5. Kingsley, L.A. et al. Lancet i, 345-349 (1987).

. Marmor, M. et al. Ann. intern. Med. 100, 809-815 (1984). 7. Lancet i, 193-194 (1986).

\section{HIV vaccination and blood} transfusion

SIR-With the increasing attention being given to the possibility of vaccination against human immunodeficiency virus (HIV $)^{1-3}$, we wish to raise the question of the impact of vaccination on blood donor screening.

Vaccination would produce seropositive individuals whose blood is reactive with $\mathrm{HIV}$-antibody screening tests currently in use for blood donor screening. As even confirmatory tests could not establish whether a positive test was the result of 